\title{
Particle Effects on Heart-Rate Regulation in Senescent Mice
}

\author{
Clarke G. Tankersley \\ Department of Environmental Health Sciences, Johns Hopkins University, Bloomberg School of Public \\ Health, Baltimore, Maryland, USA
}

Matthew Campen

Lovelace Respiratory Research Institute, Albuquerque, New Mexico, USA

\author{
Alexis Bierman, Susan E. Flanders \\ Department of Environmental Health Sciences, Johns Hopkins University, Bloomberg School of Public \\ Health, Baltimore, Maryland, USA
}

\author{
Karl W. Broman \\ Department of Biostatistics, Johns Hopkins University, Bloomberg School of Public Health, Baltimore, \\ Maryland, USA

\section{Richard Rabold} \\ Department of Environmental Health Sciences, Johns Hopkins University, Bloomberg School of Public \\ Health, Baltimore, Maryland, USA
}

\begin{abstract}
Because epidemiology studies consistently identify the elderly at risk for air pollution-related morbidity and mortality, we developed a model of senescent-dependent susceptibility based on indices of physiological aging. In the current study, we hypothesized that heart-rate regulation during particulate matter (PM) exposure differs with senescence-dependent susceptibility owing to variation in autonomic nervous control. Heart rate (HR) and heart-rate variability (HRV) parameters were measured from 162 samples of 2-min electrocardiograph (ECG) recordings in age-matched healthy $(n=5)$ and terminally senescent $(n=3)$ AKR mice during 3-h exposures to filtered-air (FA, day 1) and carbon black (CB, day $\left.4 ;<200 \mu \mathrm{g} / \mathrm{m}^{3}\right)$. On day 1 , HR was significantly $(p<.01)$ depressed during FA in terminally senescent mice. By day 4 , HR was further slowed significantly $(p<.01)$ due to the effects of $\mathrm{CB}$ exposure for 3 days. The combined effects of terminal senescence and CB exposure acted to depress HR to an average $( \pm S E M)$ $445 \pm 40 \mathrm{bpm}$, or $\sim 80 \mathrm{bpm}$ lower compared to healthy HR responses. The change in rMSSD, an HRV parameter corresponding to relative influences of parasympathetic tone on $H R$, was significantly $(p<.01)$ greater on day 1 and day 4 in terminally senescent mice compared to healthy mice. In contrast, the $\mathrm{LF} / \mathrm{HF}$ ratio, an HRV parameter derived from spectral analysis indicating relative changes in cardiac sympathetic tone, was significantly $(p<.01)$ depressed in terminally senescent mice on day 1 . By day 4 , significant increases in $\mathrm{LF} / \mathrm{HF}$ were evident in healthy mice during CB exposure, suggesting that HR regulation was associated with an increase in sympathetic tone. Alternatively, terminally senescent mice appeared to modulate a lower HR without change in $\mathrm{LF} / \mathrm{HF}$ ratio during $\mathrm{CB}$ exposure, suggesting an absence of sympathetic tone. In conclusion, older healthy mice increase cardiac sympathetic tone during PM exposure while terminally senescent mice show a greater PM-induced parasympathetic tone in regulating HR. The significance of the current results suggest that PM-induced HR regulatory changes may ultimately depend on the degree of physiological aging.
\end{abstract}

Received 3 July 2003; sent for revision 5 August 2003; accepted 15 October 2003.

This study was supported by the Electric Power Research Institute (WO8203-01) and National Institutes of Health, grant AG-21057.

Address correspondence to Clarke G. Tankersley, Division of Physiology, Bloomberg School of Public Health, Johns Hopkins University, 615 N. Wolfe St., Baltimore, MD 21205, USA.E-mail: drclarke@welchlink.jhu.edu 
Plausible biological mechanisms to explain the association between daily fluctuations in ambient air pollution and increased mortality rates remain elusive (Borja-Aburto et al., 1998). Epidemiology studies consistently identify aging and cardiopulmonary illness as risk factors in this association (Goldberg et al., 2001). Airborne particulate matter (PM) has been identified as the constituent of air pollution that is most culpable in correlating with increased cardiac mortality rates (Borja-Aburto et al., 1998; de Hartog et al., 2003; Peters et al., 2001; Stein et al., 1998). Our group and others postulate that individuals at risk to adverse cardiac effects during acute PM exposure are identified by homeostatic instability owing to physiological aging and disease (Goldberg et al., 2000; Frank \& Tankersley, 2002). We acknowledge that the lung is the target organ of air pollutant exposure, but adverse cardiac effects lead to increased mortality risk. Hence, this article implicates a dual role of PM and physiological aging in adversely altering the autonomic neural regulation of HR (Godleski et al., 2000; Pope et al., 1999; Liao et al., 1999).

Physiological aging has been defined in recent animal studies by dysfunction in vital organ systems that forecast imminent death more accurately than chronological age (McDonald et al., 1996, 1999; Tankersley et al., 2003; Frank \& Tankersley, 2002). While the assesment of physiological aging may differ among various rodent models, the inability to maintain vital regulatory mechanisms during a period of terminal senescence appears to be a common feature across species. For example, homeostatic instability associated with terminal senescence has been suggested in rodents demonstrating abrupt body weight loss and disintegration of heart rate and temperature regulation. In one study, precipitous body weight loss occurred in AKR/J mice 5-6 wk prior to death. Failure to maintain body weight coincided with decrements in average deep-body temperature and circadian rhythm disintegration in activity and heart rate (HR) (Tankersley et al., 2003). These pathophysiological changes occurred independently of chronological age.

Several potential neural mechanisms for PM-induced cardiac dysfunction have been suggested recently by Stone and Godleski (1999). Evidence from human (e.g., Liao et al., 1999; Pope et al., 1995, 1999; Peters et al., 2001) and animal studies (e.g., Watkinson, et al., 1998; Kodavanti et al., 2000) demonstrate the potential pathophysiologic mechanisms, including both tachycardic and bradycardic responses, that lead to PM-induced risk of mortality (Watkinson et al., 1998; Liao et al., 1999; Peters et al., 2001). In one scenario suggested by Stone and Godleski, ambient PM causes a generalized sympathetically induced stress response. Heart-rate increases and heart-rate variability (HRV) decreases, while an increased risk of tachyarrhythmia and fibrillation is hypothesized. In an alternative scenario, ambient PM stimulates irritant receptors of the upper airway, and vagally influences HR and HRV. The risk of bradyarrhythmia is anticipated in this case. Our postulate incorporates the possibility that the degree of physiological ag- ing may influence compensatory mechanisms, which function to defend the heart during PM exposure.

The purpose of the current study is to investigate acute PMinduced cardiac effects in terminally senescent mice that are mediated through changes in the autonomic neural control of the heart as indicated by specific changes in HRV. We hypothesize that specific PM-induced changes in HRV are dependent on the degree of physiological aging; that is, HRV responses in terminal senescence differ from responses in healthy senescence. We elected to use carbon black (CB) in this series of experiments because it represents the carboneous core of PM. Moreover, the pulmonary effects of CB are not likely attributable to overt toxicological responses. Our target $\mathrm{CB}$ concentration $\left(150 \mu \mathrm{g} / \mathrm{m}^{3}\right)$ is based on the National Air Quality Standards for a maximal 24-h average for $\mathrm{PM}_{10}$ levels. The results of the current study suggest that PM-induced changes in HR and HRV do indeed differ between healthy and terminally senescent animals.

\section{METHODS}

\section{Animals}

Inbred mice of the AKR/J strain were obtained from Jackson Laboratories (Bar Harbor, ME) as retired breeders at $\sim 180$ days of age. The mice were individually housed in an animal facility at Johns Hopkins Bloomberg School of Public Health. All animals were provided water and feed (Agway Pro-Lab RMH 1000) ad libitum. The light/dark phases of the animal facility were controlled by a 12-h cycle. All animal protocols were reviewed and approved by the Animal Care and Use Committee of the Johns Hopkins Medical Institutions.

The AKR/J strain is relatively short-lived compared with other inbred strains (Teramoto et al., 1994). The mean life span for the AKR/J strain in our facilities is $319 \pm 14$ days with a range between 197 and 548 days (Tankersley et al., 2003). Body weight gradually increases or remains stable in older healthy AKR/J mice. However, at $\sim 5$ wk prior to the end of life, an abrupt decline in body weight of $>1.5 \mathrm{~g}$ occurs between weekly measurements. This abrupt decline in body weight coincides with deficiencies in other vital physiological systems including heart rate and temperature regulation (Tankersley et al., 2003).

\section{Surgical Procedure}

The surgical procedure and the specifications surrounding the transmitter implant (model TA10ETA-F20) and a radiotelemetry system (Data Sciences International, St. Paul, $\mathrm{MN}$ ) used to measure HR and sample ECG recordings are described elsewhere (Tankersley et al., 2003). Briefly, the implant surgery was initiated by obtaining the animal's presurgical weight and anesthetizing each animal with a mixture of acepromazine $(0.5 \mathrm{ml}$ at $10 \mathrm{mg} / \mathrm{ml})$ and ketamine $(5 \mathrm{ml}$ at $100 \mathrm{mg} / \mathrm{ml})$ at a dose of $\sim 2 \mu \mathrm{l} / \mathrm{g}$. The hair covering the abdomen and chest wall was removed, betadine was applied to the exposed region of skin, and a sterile field was established surrounding the animal. 
A midline incision was made to open the abdomen, and the transmitter was inserted and sutured to the abdominal muscle. The negative ECG lead was guided through the muscle and directed subcutaneously to the right shoulder. The positive ECG lead, also guided through the muscle, was directed laterally and positioned $\sim 1 \mathrm{~cm}$ below the rib cage. Both leads were sutured to secure a lead placement resembling Lead II in traditional human ECGs. Surgery was completed within $30 \mathrm{~min}$, and recovery from anesthesia generally occurred within 60-90 min. Each animal was allowed to recover for at least $2 \mathrm{wk}$ prior to the start of data collection.

\section{PM Exposure and Assessment}

The CB (Regal 660; density $1.95 \mathrm{~g} / \mathrm{cm}^{3}$; specific surface area $112 \mathrm{~m}^{2} / \mathrm{g}$; empirical formula $\mathrm{C}_{910} \mathrm{H}_{34} \mathrm{O}_{10}$; composition $96.90 \%$ carbon, $1.42 \%$ oxygen, $0.30 \%$ hydrogen) aerosol was generated by a Wright dust feed particle generator (BGI, Inc., Waltham, MA). Following a control day of filtered-air exposure (day 1), the $\mathrm{CB}$ exposure period was initiated and maintained for $3 \mathrm{~h} /$ day for 3 consecutive days (days 2-4). During each 3-h exposure, animals were individually placed in a holding chamber designed to avoid potential stress of restraint posed by nose-only inhalation devices. The average profiles for the number and mass concentrations of the CB aerosol were measured using an aerodynamic particle sizer (APS, TSI, Atlanta, GA) and are shown in Figure 1. While the $\mathrm{CB}$ aerosol generated by this method was polydispersed in terms of particle size, the majority of particles generated (i.e., number concentration) were in the respirable range (fine mode: 0.1 to $1.0 \mu \mathrm{m}$ ). A time-weighted average of carbon black concentration was determined by gravimetric sampling at alternative exposure ports using a 25-mm diameter, $0.45-\mu \mathrm{m}$ pore size membrane filter. The flow rate through the filter was maintained in the range of $200-300 \mathrm{~cm}^{3} / \mathrm{min}$ using a critical orifice, and the weight of the filters before and after sampling was determined using a Cahn-30 microbalance (Jakab et al., 1996). From a collection of 71 gravimetric samples, the average concentration was $160 \pm 22 \mu \mathrm{g} / \mathrm{m}^{3}$.

\section{Heart-Rate Variability Analysis}

Beat-to-beat intervals were measured from 2-min ECG samples obtained every 10 min during a 3 -h exposure protocol using a peak detect algorithm created by Data Sciences. Peak detection was achievable for ECG samples lacking motion or bodyposition artifacts. The resulting tachograms (peak interval vs. time) were examined individually to detect and correct errors in intervals associated with arrhythmias or missed peak detections. To determine the frequency spectrum, each tachogram was then transformed using a Lomb-type periodogram (Press et al., 1996) and software developed in association with Dr. William P. Watkinson at the U.S. Environmental Protection Agency. This method was preferable over traditional Fourier analysis because the Lomb-type transform was advantageous for analyzing discrete data series as opposed to continuous waveforms. HRV parameters in the time domain consisted of average HR, standard deviation of R-R intervals (SDNN), and the square-root mean of the squared differences between adjacent R-R intervals (rMSSD). HRV parameters in the frequency domain were estimated using low- and high-frequency ranges. The low-frequency (LF) range was calculated as the area under each density curve from 0.2 to $1.5 \mathrm{~Hz}$, and the high-frequency (HF) range was calculated between $1.5 \mathrm{~Hz}$ and the Nyquist frequency (heart rate frequency divided by 2 , typically between 4 and $5 \mathrm{~Hz}$ during inactive periods).

\section{Data Analysis}

The data reported in the figures and tables were expressed as means $( \pm$ SEM). HR and HRV responses during $\mathrm{CB}$ exposure (day 4) were compared to similar responses obtained in the same animal during FA exposure (day 1). In addition, CB and FA responses were compared between healthy $(n=5$ mice $)$ and

B.

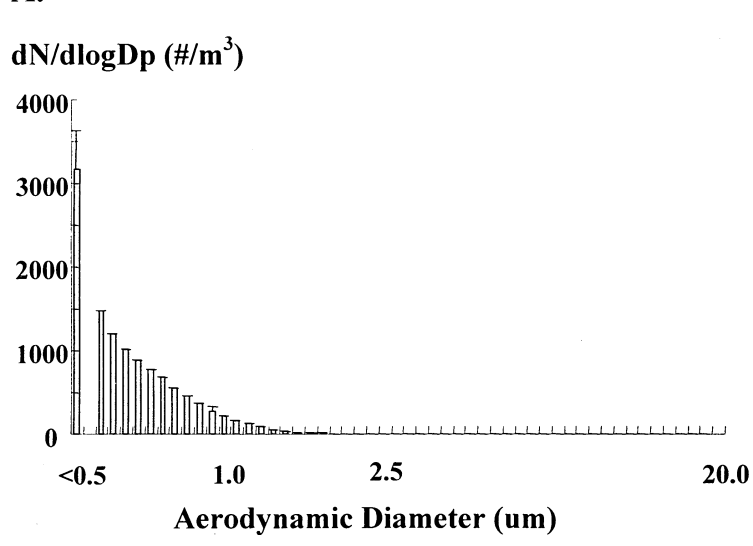

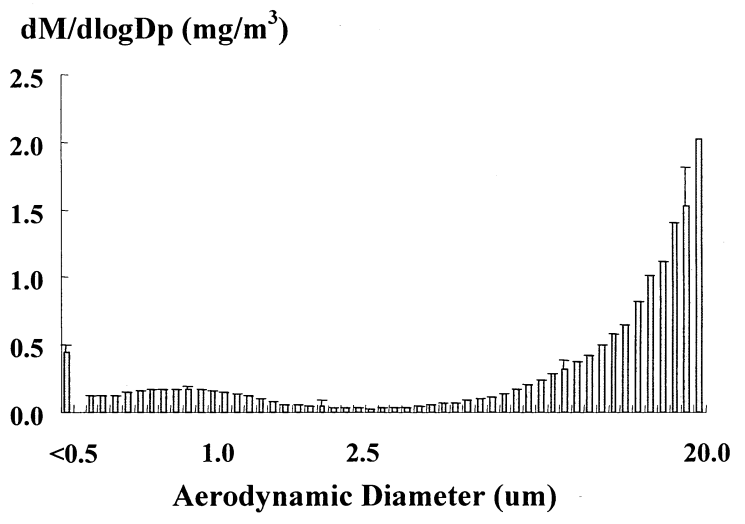

FIG. 1. Using an aerodynamic particle sizer to measure particle size, exposure assessment results show the average profile of number and mass concentration for the carbon black aerosol generated from a Wright dust feed particle generator. While the mass is dominated by particles $>10 \mu \mathrm{m}$, the majority of the number concentration is $<1.0 \mu \mathrm{m}$. 
TABLE 1

Homeostatic indices to characterize the degree of physiological aging of healthy and terminally senescent mice

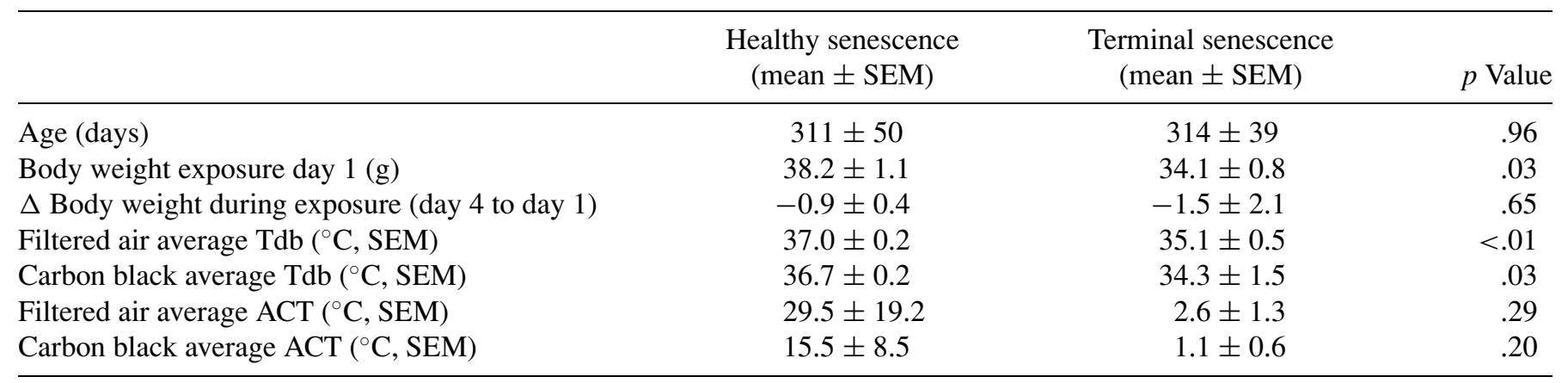

Note. Tdb, deep-body temperature; ACT.

terminally senescent ( $n=3$ mice) groups. A two-way analysis of variance (ANOVA) was performed using HR and each HRV parameter to test the significance of treatment and senescence effects. An $\alpha$ level of $p<.05$ was used to establish statistical significance.

\section{RESULTS}

Table 1 outlines several homeostatic indices used to characterize healthy and terminally senescent mice. The two groups of mice did not differ with respect to age, but each of the terminally senescent mice showed a fall in body weight of $>1.5 \mathrm{~g}$ between weekly measurements, resulting in a significantly $(p<.05)$ lower body weight in the terminally senescent group compared with healthy mice. In addition, terminally senescent mice demonstrated a significantly $(p<.05)$ lower deep-body temperature $\left(T_{\mathrm{db}}\right)$ relative to healthy mice during both FA and CB exposures.

Figure 2 illustrates three pairs of representative traces of beat-to-beat HR versus time obtained from one healthy and two terminally senescent mice. These representative samples were obtained during the last hour of FA or CB exposure. In healthy senescence (top panel), average HR fell approximately $100 \mathrm{bpm}$ between FA and CB exposures. HRV parameters in the time domain increased $\sim 56 \%$ for SDNN and $\sim 87 \%$ for $\mathrm{rMSSD}$. In contrast, there were variable HR and HRV responses in terminally senescent mice. In one case (middle panel), average HR fell 145 bpm between FA and CB exposures, which was associated with a threefold increase in SDNN and a fixed rMSSD. In an alternative case (bottom panel), average HR remained relatively stable between FA and CB exposures, but the fixed HR was accompanied by a six- to eightfold increase in SDNN and rMSSD.

Figure 3 depicts time-dependent HR changes in healthy and terminally senescent mice during FA and CB exposure. On day 1 , healthy mice showed a progressively slowing HR during FA exposure, presumably due to chamber acclimation and inactivity. By day 4, HR responses were further reduced in healthy mice at each stage of the $\mathrm{CB}$ exposure compared with FA responses. Terminally senescent mice demon- strated a significantly $(p<.01)$ reduced average HR relative to healthy mice at each stage of the FA exposure. During CB exposure, HR responses in terminally senescent mice were significantly $(p<.01)$ reduced compared to healthy HR responses. The group differences in HR between healthy and terminally senescent mice suggested that the combined effects of terminal senescence and CB exposure influenced a depression in HR to life-threatening levels (i.e., below $450 \mathrm{bpm}$ on average).

In Figures 4-6, HRV parameters illustrate group differences in SDNN, rMSSD, and the LF/HF ratio. On day 1, terminally senescent mice showed a significantly $(p<.01)$ greater rMSSD relative to healthy mice, while there was no significant effect of senescence on SDNN (Figures 4 and 5). By day 4 , both healthy and terminally senescent groups demonstrated significant $(p<.01)$ increases in SDNN and rMSSD, but the increase in rMSSD seen in terminally senescent mice was significantly $(p<.01)$ amplified compared with healthy mice. The $\mathrm{LF} / \mathrm{HF}$ ratio in terminally senescent mice was not different between FA and CB exposures, but was significantly $(p<.05)$ depressed in both exposures compared with healthy mice (Figure 6). In contrast, $\mathrm{LF} / \mathrm{HF}$ responses in healthy mice were significantly $(p<.05)$ elevated in $\mathrm{CB}$ relative to $\mathrm{FA}$ exposure.

Figure 7 depicts relationships between HRV parameters (i.e. rMSSD and LF/HF ratio) and respective HR responses. The relationship between rMSSD and HR responses in healthy mice was robust $(p<.01)$ during both FA and CB exposures, suggesting that rMSSD progressively increased as HR slowed. In contrast, the same rMSSD to HR relationship in terminally senescent mice was unstable, and lacked any obvious correlation. With respect to the relationship between $\mathrm{LF} / \mathrm{HF}$ and HR responses in healthy mice, distinguishable associations were evident between FA and CB exposures. With FA exposure, the $\mathrm{LF} / \mathrm{HF}$ ratio tended to rise as HR progressively slowed; however, the opposite association occurred during CB exposure. In terminally senescent mice, no consistent association was evident between $\mathrm{LF} / \mathrm{HF}$ and $\mathrm{HR}$ responses for either FA or $\mathrm{CB}$ exposure. 


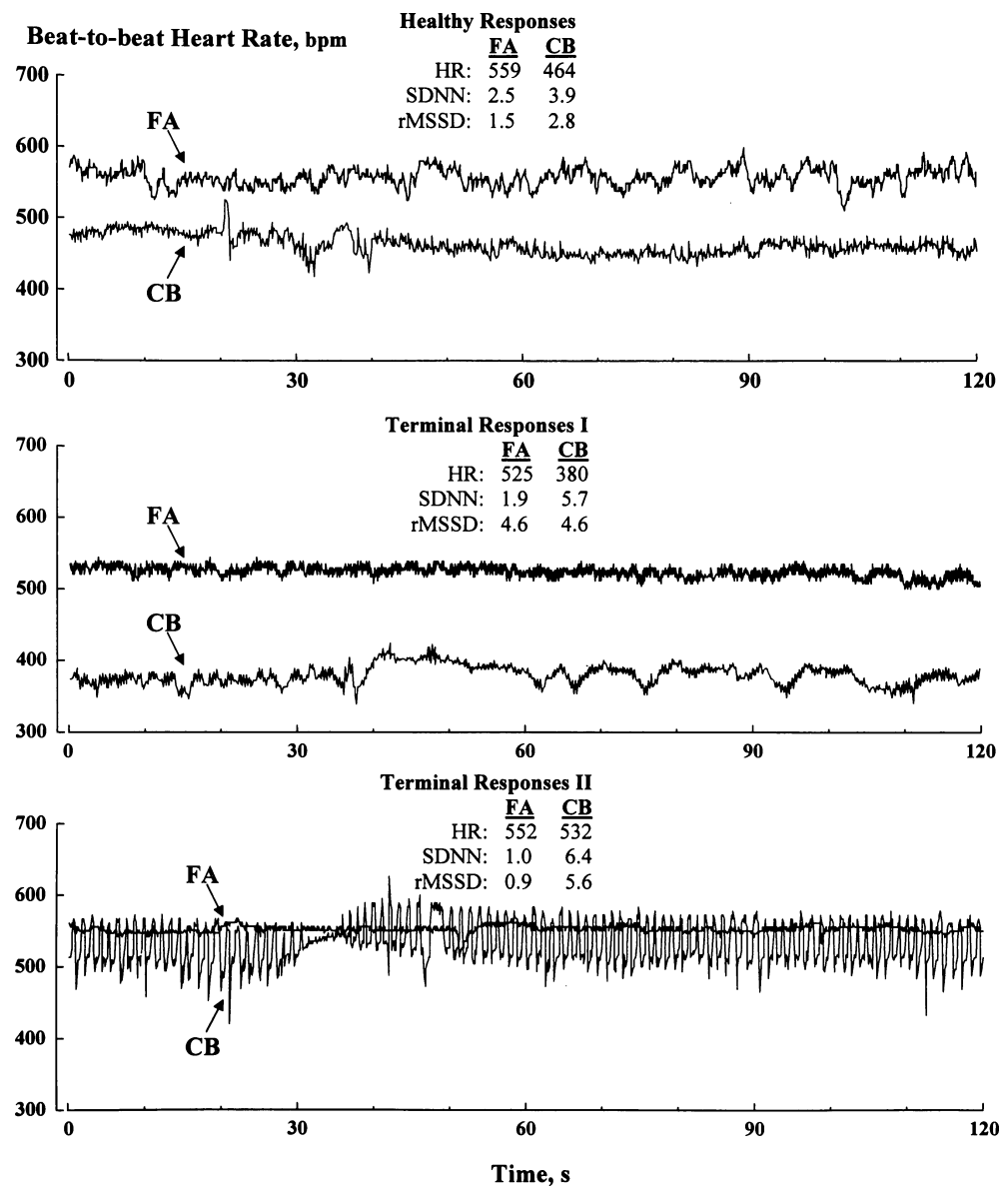

FIG. 2. Representative traces of beat-to-beat heart rate (HR) responses from healthy and terminally senescent mice. Sample data were obtained from 2-min ECG recordings to determine the R-R interval (transformed to HR) as a function of time. In the respective summary tables, average HR and heart-rate variability parameters (i.e., SDNN and rMSSD) are reported for each representative trace acquired during either filtered air (left data column) or carbon black (right data column) exposure.

\section{DISCUSSION}

The results from the current study suggest that HR and HRV parameters are acutely altered by $\mathrm{CB}$ exposure, which is an observation consistent with PM studies in humans and other animal models. The unique aspect of our findings demonstrates that the characteristics of acute PM-induced changes in HR and HRV are dependent on the degree of physiological aging. We have established an inventory of homeostatic indices that are used to define different stages of aging and to distinguish a period of terminal senescence. During this period, an abrupt loss in body weight occurs coincident with the disintegration in heart rate and temperature regulation (Tankersley et al., 2003). Using this model of aging, PM exposure during terminal senescence leads to an exaggerated bradycardia, which is accompanied by increases in both SDNN and rMSSD. This acute PM-induced change in HRV profile suggests that the exaggerated bradycardia results from a predominantly greater parasympathetic tone in regulating HR. Moreover, the observation that the LF/HF ratio remains unchanged in terminally senescent mice during PM exposure alludes to the absence of any significant contribution in sympathetic tone. While inferences regarding PM-induced changes in the autonomic neural control of HR require additional studies, it is clear from the relationship between rMSSD and HR that altered HR regulation during acute PM exposure was more varied and less predictable in terminally senescent mice.

The representative traces in Figure 2 are illustrative of the general inferences drawn from our results. In the top panel, a lower HR and modest increases in SDNN and rMSSD characterize healthy senescent responses to $\mathrm{CB}$ exposure compared with FA responses. These changes in HR regulation are likely associated with chamber acclimation and exposure to PM. The relationship between rMSSD and HR (Figure 7) in healthy senescent mice suggests that slowing HR responses during both FA and CB exposure are mediated by an increased parasympathetic tone. Likewise, the negative relationship between $\mathrm{LF} / \mathrm{HF}$ ratio and HR (Figure 7) during FA exposure alludes to a potential increased sympathetic tone as HR slows, while the inverse 

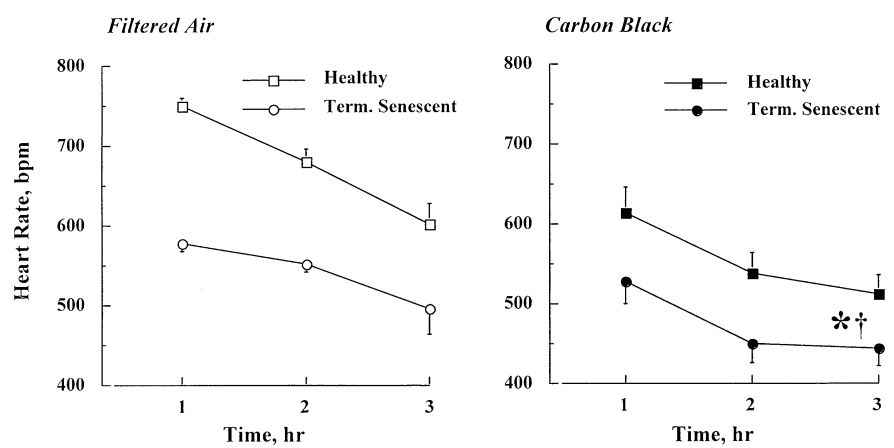

FIG. 3. Average heart rate responses as a function time during 3-h exposures to either filtered air (FA; day 1) or carbon black (CB; day 4) in healthy and terminally senescent mice. In both groups of mice, a progressively decreasing HR occurs during FA, presumably due to chamber acclimation and inactivity. Exposure to CB leads to a greater slowing of HR in both groups, but the PM-induced bradycardic responses are amplified in terminally-senescent mice. Asterisk indicates significance at $p<.01$ for healthy vs. terminally senescent, and dagger indicates significance at $p<.01$ for filtered air vs. carbon black.

relationship during $\mathrm{CB}$ exposure indicates a likely decrease in sympathetic tone. One pitfall in the latter interpretation is related to a technical inability to measure beat-to-beat variability at extremely high heart rates (e.g., >750 bpm). Nevertheless, the results suggest that both branches of the autonomic neural control of HR are activated at varying degrees by PM exposure in healthy senescent mice. This predictable pattern in healthy senescent mice exposed to PM is not evident in terminally senescent mice.
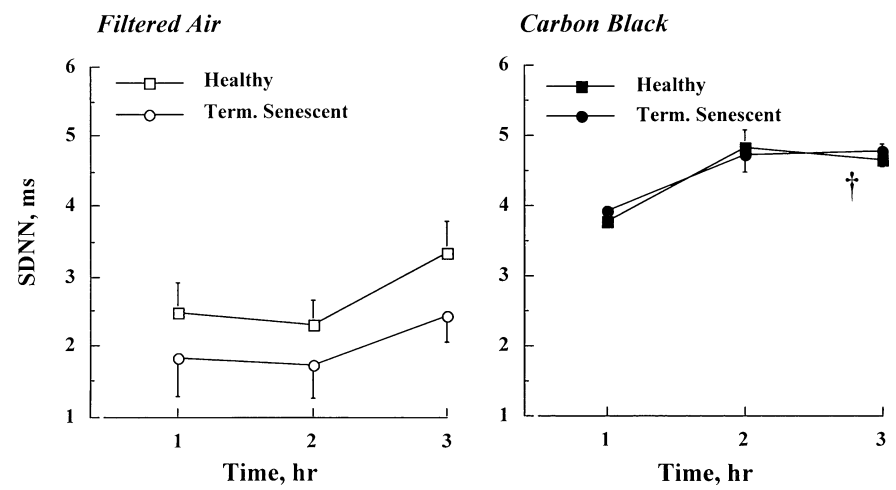

FIG. 4. Total heart-rate variability in the time domain (SDNN) as a function of time during 3-h exposures to either filtered air (FA; day 1) or carbon black (CB; day 4) in healthy and terminally senescent mice. While there is no apparent senescence effect on SDNN, CB exposure resulted in a significant increase in SDNN in both groups. Dagger indicates significance at $p<.01$ for filtered air vs. carbon black.
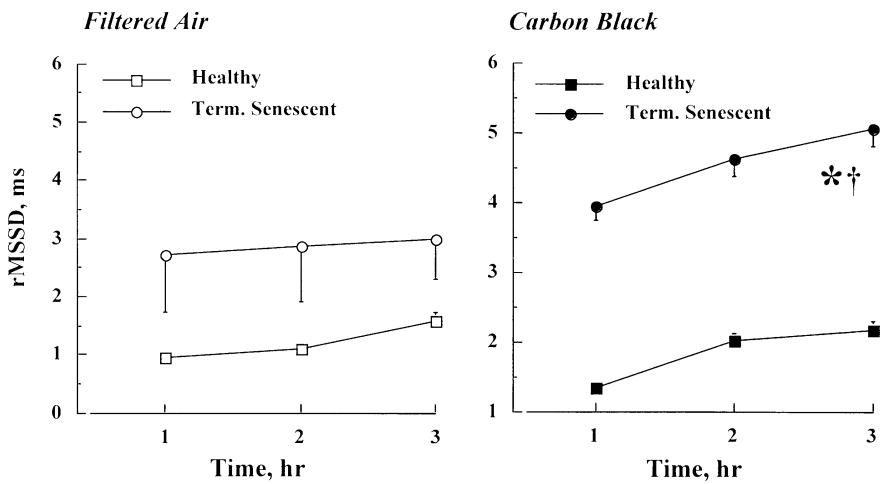

FIG. 5. Beat-to-beat heart-rate variability in the time domain (rMSSD) as a function of time during 3-h exposures to either filtered air (FA; day 1) or carbon black (CB; day 4) in healthy and terminally senescent mice. In general, rMSSD was significantly influenced by both the effects of terminal senescence and $\mathrm{CB}$ exposure. Because rMSSD represents an indicator of changes in parasympathetic regulation of $\mathrm{HR}$, the results suggest that terminally senescent mice demonstrate greater parasympathetic input compared with healthy mice. Moreover, CB exposure further exaggerates the group differences in parasympathetic regulation of $\mathrm{HR}$. Asterisk indicates significance at $p<.01$ for healthy vs. terminally senescent, and dagger $(p<.01)$ for filtered air vs. carbon black.
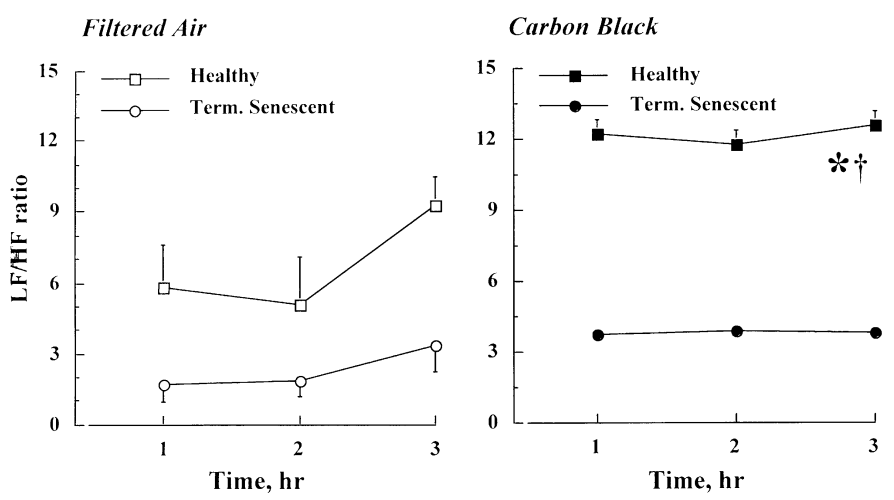

FIG. 6. Heart rate variability in the frequency domain, as represented by the low-frequency to high-frequency ratio (LF/HF ratio), shown as a function of time during 3-h exposures to either filtered air (FA; day 1) or carbon black (CB; day 4) in healthy and terminally senescent mice. In general, the LF/HF ratio was significantly influenced by both the effects of terminal senescence and $\mathrm{CB}$ exposure. Because the LF/HF ratio represents an indicator of changes in sympathetic regulation of $\mathrm{HR}$, the results suggest that healthy mice demonstrate greater sympathetic input compared with terminally-senescent mice. Moreover, $\mathrm{CB}$ exposure further exaggerates the group differences in sympathetic regulation of HR. Asterisk indicates significance at $p<.01$ for healthy vs. terminally senescent, and dagger $(p<.01)$ for filtered air vs. carbon black. 

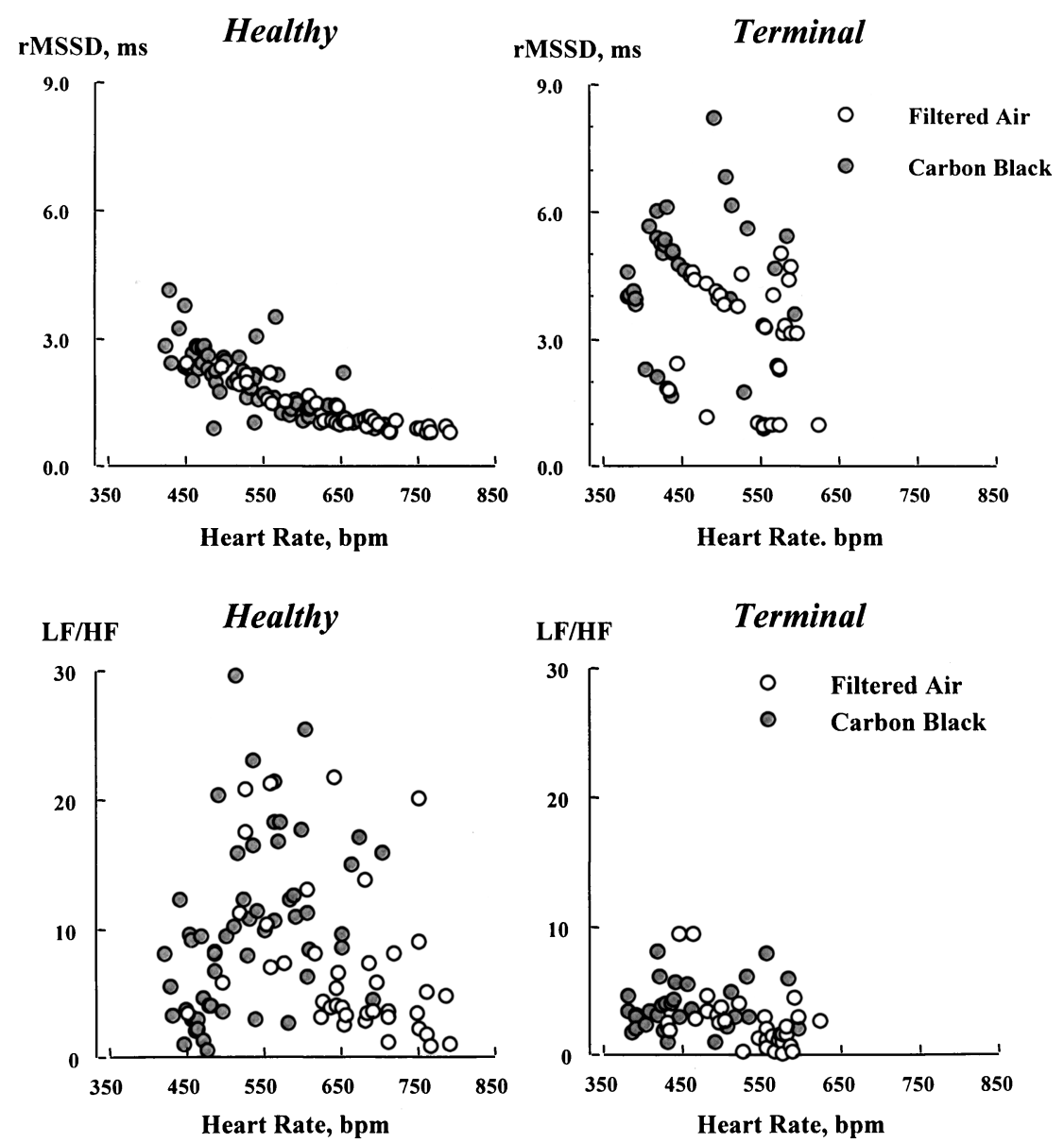

FIG. 7. Relationships between heart-rate variability parameters (rMSSD and LF/HF ratio) and heart responses (HR) for healthy and terminally senescent mice exposed to filtered air (FA; day 1) or carbon black (CB; day 4). As an index of PM-induced parasympathetic tone, the relationship between rMSSD and HR is robust for healthy senescent mice during both FA and CB exposures; however, the same relationship is quite variable in terminally senescent mice. The relationship between the LF/HF ratio and HR suggests that sympathetic tone is modified during both FA and CB exposures in healthy mice, and is virtually absent in terminally senescent mice.

One profile of HR and HRV responses demonstrated in terminally senescent mice is represented in the middle panel of Figure 2. In this case, the HR responses are initially lower during FA, and are markedly depressed during CB exposure (e.g., $<400$ bpm). The PM-induced bradycardia is accompanied by an increased SDNN due, in part, to a modestly greater $\mathrm{LF} / \mathrm{HF}$ ratio (Figure 7). While rMSSD responses are elevated relative to healthy responses, this particular HRV parameter remains relatively stable between FA and CB exposures. One potential explanation for this distinctive profile suggests that parasympathetic tone modulates HR in the absence of any substantial sympathetic tone, as indicated by relatively small changes in the $\mathrm{LF} / \mathrm{HF}$ ratio (Figure 7 ). The exaggerated $\mathrm{PM}$-induced bradycardia may also imply that other HR regulatory mechanisms are altered in terminally-senescent mice. An alternative profile of HR and HRV responses demonstrated by terminally senescent mice is illustrated in the bottom panel of Figure 2. In this case, HR responses during FA exposure are similar to healthy responses, but HRV parameters, including SDNN and rMSSD, are notably depressed. With CB exposure, the beat-to-beat variation reveals a unique pattern that is identified by $\mathrm{HR}$ oscillations $(<1.0 \mathrm{~Hz})$ accompanied by an inordinate elevation in HRV parameters. Keeping in mind that PM-induced bradycardia is observed in the previously illustrated representative traces, a regulatory mechanism to explain these particular HR oscillations remains uncertain. One possible mechanism suggests that blood pressure regulation is compromised with PM-induced bradycardia, and HR is periodically accelerated through baroreceptor activity; however, this PM-induced cardiovascular mechanism requires further study.

Several epidemiologic studies have explored the association between ambient air pollution exposure and changes in the 
autonomic regulation of HR in humans (Dockery et al., 999; Pope et al., 1999a, 1999b; Gold et al., 2000; Liao et al., 1999; Peters et al., 2001). Two studies (Gold et al., 2000; Liao et al., 1999) suggested that high-frequency HRV declines in association with higher levels of PM exposure. In contrast, a third study (Pope et al., 1999) demonstrated increased high-frequency HRV in the short term, while low-frequency HRV decreased. Because changes in HRV forecast susceptibility to cardiac morbidity and mortality (Bigger et al., 1995; Fei et al., 1994; Tsuji et al., 1994, 1996; Lanza et al., 1998; Algra et al., 1993; Ponikowski et al., 1997), some resolution to this controversy is critical. Reduced $\mathrm{HRV}$, for example, has been used to predict increased risk of adverse cardiac events including sustained ventricular tachycardia (Fei et al., 1994). However, it remains unclear whether or not altered HRV is mechanistically linked to increased risk of cardiac mortality associated with human exposure to PM. Complementary animal studies have also investigated the adverse cardiac effects of acute PM exposure (Godleski et al., 2000; Watkinson et al., 2001; Kodavanti \& Costa, 2001; Kodavanti et al., 1998, 2000). In terms of PM-induced tachycardia, studies exposing rats to known components of ambient PM have demonstrated cardiac arrhythmias (Watkinson et al., 1998) and other stress-induced regulatory changes (Killingsworth et al., 1997). With respect to PM-induced bradycardia, cardiopulmonary responses to hypoxemia, such as pulmonary hypertension, may be one aspect of the ensuing compromise in cardiac function (Watkinson et al., 1998). Collectively, current and previous studies suggest that autonomic neural control of HR is acutely altered by PM exposure resulting in chaotic adjustments between sympathetic and parasympathetic nervous activity.

Some resolution to equivocal findings linking HRV changes to PM-induced mortality risk may depend on the integrity of multiple physiological systems and the degree of homeostatic competence during exposure to poor air quality. In older healthy individuals, many of the compensatory mechanisms remain accessible to defend vital organ systems. In the current study, results from healthy senescent mice demonstrate a modest slowing of the heart in response to CB exposure. The HRV parameters are also altered in healthy mice during $\mathrm{CB}$ exposure. These changes include increases in both SDNN and the LF/HF ratio coincident with only a modest increase in rMSSD. This altered HRV profile is likely an indicator of increased sympathetic tone in regulating HR, and as suggested by Stone and Godleski (1999), is derived from a generalized stress-induced increase in sympathetic neural output; however, it is contrary to the compensation that occurs in terminally-senescent mice. Previous work using the AKR/J inbred mouse strain as a model of physiological aging demonstrated discrete stages of disintegration in multiple systems, which resulted in a predictable sequence of pathophysiological events occurring with imminent death (Tankersley et al., 2003). These results showed instability in cardiac regulatory mechanisms distinguished by an abrupt decline in the daily average HR, which occurred 3-4 wk before death. Based on these previous findings, we estimate that this group of terminally senescent mice was exposed to $\mathrm{CB}$ 3-4 wk prior to death. During this period, compensatory mechanisms available to defend the heart during $\mathrm{CB}$ exposure leads to HR reductions mediated predominantly by enhanced parasympathetic tone without significant changes in sympathetic input.

One hypothesis available from epidemiological literature suggests that individuals susceptible to PM-induced mortality are the elderly and patients with cardiopulmonary disease, who are generally characterized by extreme fragility (Goldberg et al., 2000, 2001; Frank \& Tankersley, 2002). The adverse cardiac effects in vulnerable individuals may differ from those individuals identified as homeostatically competent, that is, having multiple and parallel defense mechanisms available. In this regard, the current results support a plausible explanation for PM-induced mortality risk by demonstrating variable HR regulatory mechanisms in response to PM exposure that are dependent on the degree of physiological aging. Whether or not these results can be demonstrated in human populations may require an individual assessment of physiological aging. Furthermore, a greater understanding of the differences in the autonomic regulation of HR between humans and mice may impact the translation to epidemiological results. Lastly, the CB exposure used in the current study may not necessarily reflect the carbon composition in ambient PM.

In conclusion, the adverse cardiac effects of PM involve different changes in HR regulation depending on the extent of individual fragility or the degree of physiological aging. The current results support the likelihood that both sympathetic and parasympathetic tone are altered during PM exposure in healthy senescent mice. Alternatively, adjustments in parasympathetic tone dominate HR regulation during acute PM exposure in terminally senescent mice. Future studies are required to test these postulations more directly.

\section{REFERENCES}

Algra, A., Tijssen, J. G., Roelandt, J. R., Pool, J., and Lubsen, J. 1993. Heart rate variability from 24-hour electrocardiography and the 2-year risk for sudden death. Circulation 88:180-185.

Bigger, J. T. J., Fleiss, J. L., Steinman, R. C., Rolnitzky, L. M., Schneider, W. J., and Stein, P. K. 1995. RR variability in healthy, middle-aged persons compared with patients with chronic coronary heart disease or recent acute myocardial infarction. Circulation 91:1936-1943.

Borja-Aburto, V. H., Castillejos, M., Gold, D. R., Bierzwinski, S., and Loomis, D. 1998. Mortality and ambient fine particles in southwest Mexico City, 1993-1995. Environ. Health Perspect. 106:849855.

de Hartog, J. J., Hoek, G., Peters, A., Timonen, K. L., Ibald-Mulli, A., Brunekreef, B., Heinrich, J., Tiittanen, P., van Wijnen, J. H., Kreyling, W., Kulmala, M., and Pekkanen, J. 2003. Effects of fine and ultrafine particles on cardiorespiratory symptoms in elderly subjects with coronary heart disease: The ULTRA study. Am. J. Epidemiol. 157:613-623. 
Dockery, D. W., Pope, C. A., Kanner, R. E., Martin, V. G., and Schwartz, J. 1999. Daily changes in oxygen saturation and pulse rate associated with particulate air pollution and barometric pressure. Res. Rep. Health Effects Inst. pp. 1-19.

Fei, L., Statters, D. J., Anderson, M. H., Malik, M., and Camm, A. J. 1994. Relationship between short- and long-term measurements of heart rate variability in patients at risk of sudden cardiac death. Pacing Clin. Electrophysiol. 17:2194-2200.

Frank, R., and Tankersley, C. 2002. Air pollution and daily mortality: A hypothesis concerning the role of impaired homeostasis. Environ. Health Perspect. 110:61-65.

Godleski, J. J., Verrier, R. L., Koutrakis, P., Catalano, P., Coull, B., Reinisch, U., Lovett, E. G., Lawrence, J., Murthy, G. G., Wolfson, J. M., Clarke, R. W., Nearing, B. D., and Killingsworth, C. 2000. Mechanisms of morbidity and mortality from exposure to ambient air particles. Res. Rep. Health Effects Inst. pp. 5-88.

Gold, D. R., Litonjua, A., Schwartz, J., Lovett, E., Larson, A., Nearing, B., Allen, G., Verrier, M., Cherry, R., and Verrier, R. 2000. Ambient pollution and heart rate variability. Circulation 101:12671273.

Goldberg, M. S., Bailar, J. C., Burnett, R. T., Brook, J. R., Tamblyn, R., Bonvalot, Y., Ernst, P., Flegel, K. M., Singh, R. K., and Valois, M. F. 2000. Identifying subgroups of the general population that may be susceptible to short-term increases in particulate air pollution: A time-series study in Montreal, Quebec. Res. Rep. Health Effects Inst. pp. 7-113.

Goldberg, M. S., Burnett, R. T., Bailar, J. C., Brook, J., Bonvalot, Y., Tamblyn, R., Singh, R., Valois, M. F., and Vincent, R. 2001. The association between daily mortality and ambient air particle pollution in Montreal, Quebec. 2. Cause-specific mortality. Environ. Res. 86:26-36.

Jakab, G. J., Clarke, R. W., Hemenway, D. R., Longphre, M. V., Kleeberger, S. R., and Frank, R. 1996. Inhalation of acid coated carbon black particles impairs alveolar macrophage phagocytosis. Toxicol. Lett. 88:243-248.

Killingsworth, C. R., Alessandrini, F., Murthy, G. G. K., Catalano, P. J., Paulauskis, J. D., and Godleski, J. J. 1997. Inflammation, chemokine expression, and death in monocrotaline-treated rats following fuel oil fly ash inhalation. Inhal. Toxicol. 9:541-565.

Kodavanti, U. P., and Costa, D. L. 2001. Rodent models of susceptibility: What is their place in inhalation toxicology? Respir. Physiol. 128:57-70.

Kodavanti, U. P., Costa, D. L., and Bromberg, P. A. 1998. Rodent models of cardiopulmonary disease: Their potential applicability in studies of air pollutant susceptibility. Environ. Health Perspect. 106(suppl. 1):111-130.

Kodavanti, U. P., Schladweiler, M. C., Ledbetter, A. D., Watkinson, W. P., Campen, M. J., Winsett, D. W., Richards, J. R., Crissman, K. M., Hatch, G. E., and Costa, D. L. 2000. The spontaneously hypertensive rat as a model of human cardiovascular disease: Evidence of exacerbated cardiopulmonary injury and oxidative stress from inhaled emission particulate matter. Toxicol. Appl. Pharmacol. 164:250-263.

Lanza, G. A., Guido, V., Galeazzi, M. M., Mustilli, M., Natali, R., Ierardi, C., Milici, C., Burzotta, F., Pasceri, V., Tomassini, F., Lupi, A., and Maseri, A. 1998. Prognostic role of heart rate variability in patients with a recent acute myocardial infarction. Am. J. Cardiol. 82:1323-1328.
Liao, D., Creason, J., Shy, C., Williams, R., Watts, R., and Zweidinger, R. 1999. Daily variation of particulate air pollution and poor cardiac autonomic control in the elderly. Environ. Health Perspect. 107:521-525.

McDonald, R. B., Florez-Duquet, M., Murtagh-Mark, C., and Horwitz, B. A. 1996. Relationship between cold-induced thermoregulation and spontaneous rapid body weight loss of aging F344 rats. Am. J. Physiol. 271:R1115-R1122.

McDonald, R. B., Hoban-Higgins, T. M., Ruhe, R. C., Fuller, C. A., and Horwitz, B. A. 1999. Alterations in endogenous circadian rhythm of core temperature in senescent Fischer 344 rats. Am. J. Physiol. 276:R824-R830.

Peters, A., Perz, S., Doring, A., Stieber, J., Koenig, W., and Wichmann, H. E. 1999. Increases in heart rate during an air pollution episode. Am. J. Epidemiol. 150:1094-1098.

Peters, A., Dockery, D. W., Muller, J. E., and Mittleman, M. A. 2001. Increased particulate air pollution and the triggering of myocardial infarction. Circulation 103:2810-2815.

Ponikowski, P., Anker, S. D., Chua, T. P., Szelemej, R., Piepoli, M., Adamopoulos, S., Webb-Peploe, K., Harrington, D., Banasiak, W., Wrabec, K., and Coats, A. J. 1997. Depressed heart rate variability as an independent predictor of death in chronic congestive heart failure secondary to ischemic or idiopathic dilated cardiomyopathy. Am. J. Cardiol. 79:1645-1650.

Pope, C. A., Thun, M. J., Namboodiri, M. M., Dockery, D. W., Evans, J. S., Speizer, F. E., and Heath, C. W. J. 1995. Particulate air pollution as a predictor of mortality in a prospective study of U.S. adults. Am. J. Respir. Crit. Care Med. 151:669-674.

Pope, C. A., Dockery, D. W., Kanner, R. E., Villegas, G. M., and Schwartz, J. 1999a. Oxygen saturation, pulse rate, and particulate air pollution: A daily time-series panel study. Am. J. Respir. Crit. Care Med. 159:365-372.

Pope, C. A., Verrier, R. L., Lovett, E. G., Larson, A. C., Raizenne, M. E., Kanner, R. E., Schwartz, J., Villegas, G. M., Gold, D. R., and Dockery, D. W. 1999. Heart rate variability associated with particulate air pollution. Am. Heart J. 138:890-899.

Press, W., Teukolsky, S., Vetterling, W., and Flannery, B. 1996. Numerical recipes in $C$, The art of scientific computing, pp. 496-584. Cambridge University Press.

Stein, P. K., Nelson, P., Rottman, J. N., Howard, D., Ward, S. M., Kleiger, R. E., and Senior, R. M. 1998. Heart rate variability reflects severity of COPD in PiZ alpha1-antitrypsin deficiency. Chest 113:327-333.

Stone, P. H., and Godleski, J. J. 1999. First steps toward understanding the pathophysiologic link between air pollution and cardiac mortality. Am. Heart J. 138:804-807.

Tankersley, C. G., Irizarry, R., Flanders, S. E., Rabold, R., and Frank, R. 2003. Unstable heart rate and temperature regulation predict mortality in AKR/J mice. Am. J. Physiol. Regul. Integr. Comp. Physiol. 284:R742-R750.

Teramoto, S., Fukuchi, Y., Uejima, Y., Teramoto, K., Oka, T., and Orimo, H. 1994. A novel model of senile lung: Senescenceaccelerated mouse (SAM). Am.J. Respir. Crit. Care Med. 150:238 244.

Tsuji, H., Venditti, F. J. J., Manders, E. S., Evans, J. C., Larson, M. G., Feldman, C. L., and Levy, D. 1994. Reduced heart rate variability and mortality risk in an elderly cohort. The Framingham Heart Study. Circulation 90:878-883. 
Tsuji, H., Larson, M. G., Venditti, F. J. J., Manders, E. S., Evans, J. C., Feldman, C. L., and Levy, D. 1996. Impact of reduced heart rate variability on risk for cardiac events. The Framingham Heart Study. Circulation 94:2850-2855.

Watkinson, W. P., Campen, M. J., and Costa, D. L. 1998. Cardiac arrhythmia induction after exposure to residual oil fly ash particles in a rodent model of pulmonary hypertension. Toxicol. Sci. 41:209216.

Watkinson, W. P., Campen, M. J., Nolan, J. P., and Costa, D. L. 2001. Cardiovascular and systemic responses to inhaled pollutants in rodents: Effects of ozone and particulate matter. Environ. Health Perspect. 109(suppl. 4):539-546. 